\title{
Theoretical study of electrical contact dustiness impact on the transition resistance
}

\author{
Ekaterina Lyuminarskaja ${ }^{1}{ }^{*}$, , Igor Lyuminarsky ${ }^{1}$, and Kirill Selivanov ${ }^{1}$ \\ ${ }^{1}$ Bauman Moscow State Technical University, 105005, Moscow, Vtoraya Baumanskaya street, 5, \\ build. 1, Russian Federation
}

\begin{abstract}
The article considers a mathematical model for determination of transition resistance in electrical contacts. The suggested model considers elastic, elastoplastic and plastic deformations of micro-protrusions on rough contact surfaces, the presence of surface films and dust particles on them. A theoretical dependence of possible contact failure on dust content has been obtained.
\end{abstract}

\section{Introduction}

One of the methods to increase electronics reliability is prevention of operation faults and failures. Failures are frequently caused by faulty electrical contacts. Electrical contact faults are caused by thermal, electrical, mechanical and chemical phenomena taking place in the transition area. At present low-level contacts are widespread, i.e. contacts with less than 0.1A current and $5 \mathrm{~V}$ max voltage. Fault and failure conditions in such contacts are basically caused by dust and other contaminants in the intercontact space, formation of films, which remain in place at contact closing. Development of efficient methods for determination of failure causes in low-level electrical contacts requires creation of mathematical models that enable to determine transition area characteristics in the real operation conditions.

\section{Problem description}

The electrical contact theory is described in the foreign publications by R. Holm [1], W. Merle [2], J. Greenwood and J. Williamson [3, 4], in the works of Soviet scientists, such as B.S. Sotskov [5], A.P. Levin [6], N.K. Myshkin [7], etc. [8-19]. In all publications an electrical contact is represented as a contact between two rough surfaces. Surface protrusions constitute a multitude of contact areas electrical current passes through.

The purpose of this research is development of a refined method for determination of transition resistance in electrical contacts in the real operation conditions and theoretical study of the impact of the dust accumulated on the contact on the possibility of failure.

\footnotetext{
* Corresponding author: lyuminarskaja.caterina@yandex.ru
} 


\section{Mathematical Model}

The suggested mathematical model, as opposed to the current methods, considers the following:

- the possibility of elastic, elastoplastic and plastic deformation of individual protrusions of contact surfaces depending on the load;

- elastic deformation of the base underlaying the spherical protrusions constituting the rough surfaces;

- change of protrusion characteristics under repeated load due to plastic deformations of individual protrusions;

- the possibility of dust sedimentation on contact surfaces.

In the calculation the rough surfaces are substituted with equivalent surfaces (Fig. 1), constituted by a number of spherical segments randomly located on elastic bases. For simplification purposes it is assumed that the protrusions of the two surfaces are located opposite one another. The micro-protrusions height is considered a random value, and the radii of all protrusions are considered equal to one another.

The spherical protrusion height is determined by the relative value $\xi_{i}$ considered to be a random value for calculation purposes.

$$
\xi_{i}=\frac{p_{i}}{R_{p}}
$$

where $i$ is a protrusion number, $R_{p}$ is taper height [10].

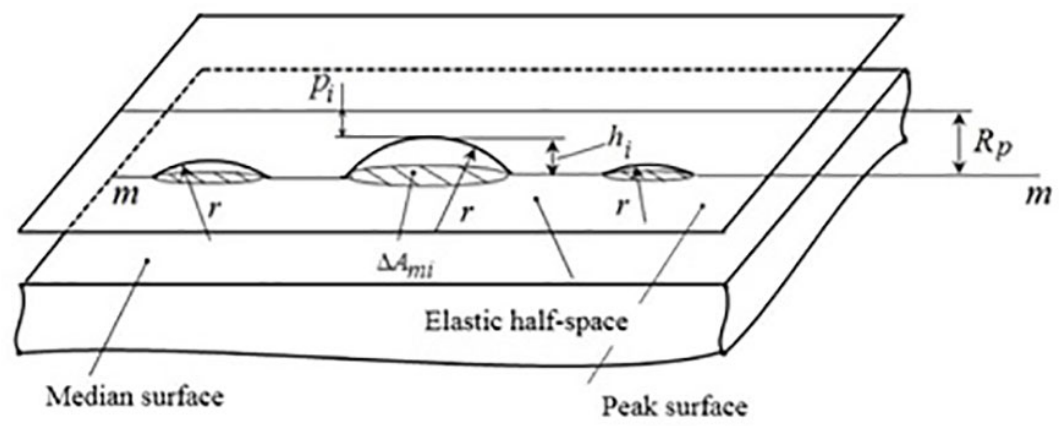

Fig. 1. Equivalent substituting surface

The distribution law $\xi$ is set on the condition that the supporting curve of the substituting equivalent surface coincides with the supporting curve of the real surface $\eta_{s}(\xi)$, normally approximated as:

$$
\eta_{s}(\xi)=t_{m} \cdot \xi^{v}
$$

Parameters of the supporting curve $t_{m}$ and $v$ depend on surface roughness [9, 12]. For the spherical protrusions the distribution law $\xi$ is defined by the expression suggested by $N$. Demkin:

$$
F(\xi)=\xi^{v-1}
$$


For calculation purposes all protrusions are segregated into three groups. The first group includes interacting protrusions, the second one - not interacting protrusions. The third group includes protrusion with dust particles in between.

The force of interaction between two micro-protrusions, the contact area radius and the average contact pressure are determined via their approximation, depending on the approximation of peak surfaces, micro-protrusion height and their movement as rigid bodies on an elastic base. If approximation of two protrusion is less than $6,7 \frac{\sigma_{T}^{2} \cdot r_{p}}{E_{p}^{2}}$, the abovementioned values are determined using the Herz Formulas, otherwise, they are determined using the formulas given in [7,9], for elastoplastic and developed plastic deformation of the spherical protrusions.

After load removal at the first switching, elastic deformations disappear, but plastic deformations are preserved. Micro-protrusion heights and radii are changed. Computational research showed that the mentioned changes should be considered for determination of transition area characteristics. Approximate formulas for determination of micro-protrusion residual height and radius values have been obtained as a result of computational research carried out using the finite elements method.

Investigational study by various authors showed that dust particles, normally of the irregular shape, are always present on contact surfaces. For computational convenience irregular dust particles are substituted with equivalent spheres.

The mathematical model suggests that interaction between two protrusions with a dust particle is of elastic nature. In this case the interaction force is determined using the Herz Formula. If at interaction the tensile stress in the dust particle center exceeds the ultimate tensile strength of the dust particle material, the particle is destroyed and does not affect the calculation.

The dust particle diameter $d_{\Pi}$ is considered a random value having a Rayleigh distribution. The average dust particle diameter in switching elements (sealed contacts, micro-switches and relays) is $10-20 \mu \mathrm{m}$.

The surface dustiness measurement unit is dust particle concentration equal to the ratio between the particle number $n_{p}^{*}$ and the area of the surface $A$ they are located on:

$$
v_{\mathrm{cp}}=\frac{n_{p}^{*}}{A}
$$

Thus, the total quantity of dust particles in a gap between contacts is equal the number of dust particles on two interacting surfaces:

$$
n_{p}=2 n_{p}^{*}=2 v_{\mathrm{cp}} A
$$

For simplification purposes it has been assumed that the dust particles are located between the peaks of the rough surfaces in a random manner. The dust particle distribution on protrusion peaks is even. The number of rough surface protrusion a dust particle is located on is determined using the formula (6):

$$
i_{p}=\chi \cdot n
$$

where $\chi$ is a random value evenly distributed in the $[0,1]$ interval; $n$ is the number of protrusion on the rough surface.

The calculation is performed as follows. At the first stage, rough surfaces are modeled with a set of spherical segments and diameters of dust particles randomly located on the contact surfaces are determined. At the second calculation stage, approximation of the contact surfaces is set for determination of micro-irregularities approximations, interaction forces 
and contact area radii of individual protrusions, dust particle deformation and the number of destroyed particles.

The essential electrical contact characteristic is transition resistance. The transition area resistance is represented as parallel connection of individual micro-protrusions resistances.

Micro-protrusion surfaces are covered with films (oxide, sulphide films, etc.). If the average contact pressure of two micro-protrusions exceeds the ultimate strength of the film, the contacting micro-protrusions resistance is conditioned only by current line concentration on the contact area $[1,2,5,6,7,9]$ and determined using the following formula:

$$
R_{p i}=\gamma \cdot \frac{\left(\rho_{1}+\rho_{2}\right)}{4 r_{a i}}
$$

where $\rho_{1}, \rho_{2}$ are specific resistance of contact materials; $r_{a i}$ is the radius of two protrusions contact area.

Resistance of two protrusions with partially destroyed films exceeds their resistance without surface films. It is considered in the calculation as the $\gamma$ factor [9].

If under the contact force action the surface films are not destroyed, the contacting protrusions resistance is constituted by contraction resistance and surface resistance of the films and determined using the following formula:

$$
R_{p i}=\frac{\rho_{1}+\rho_{2}}{4 r_{a i}}+\frac{\sigma_{1}+\sigma_{2}}{\pi \cdot r_{a i}^{2}}
$$

where $\sigma_{1}, \sigma_{2}$ are specific resistances of surface films.

\section{Theoretical stady results}

The theoretical study has been performed for spherical brass contacts with the $R_{1}=R_{2}=$ $3 \mathrm{~mm}$ radii and the $F=0,7 \mathrm{H}$ contact pressing force. The specific resistance of contact materials $\rho_{1}=\rho_{2}=68 \mathrm{microOhm} \cdot \mathrm{mm}$.

It is commonly known that at switching causes contact bounce. The maximum contact interaction force exceeds the nominal force more than 10 times. The force acts for a very short period ( $\approx 5$ microsec), which is not enough for the major deformation of the elastic element. This short impulse can destroy dust particles on contact surfaces.

In view of the above, failure condition determination was performed in two stages. At the first stage, the contacts were impacted by the force $F_{\max }=9 \mathrm{H}$. The dust particles were partly destroyed. At the second stage, the contacts were impacted by the nominal force $F_{n o m}=0,7 \mathrm{H}$, and electrical resistance was measured. Failure was registered by the transition resistance value. If the transition resistance exceeded the nominal value two and more times, a failure was registered. The calculation was performed using the Monte-Carlo method.

The calculation results are shown in Fig.2. At the dust concentration on contact surfaces $v_{\text {avg }}=20 \mathrm{~mm}^{2}$ the probability of failure reaches $15 \%$. In case of two-fold increase of dust concentration (from 25 to 50 particles per $1 \mathrm{~mm}^{2}$ ) the probability of failure increases 3 times. 


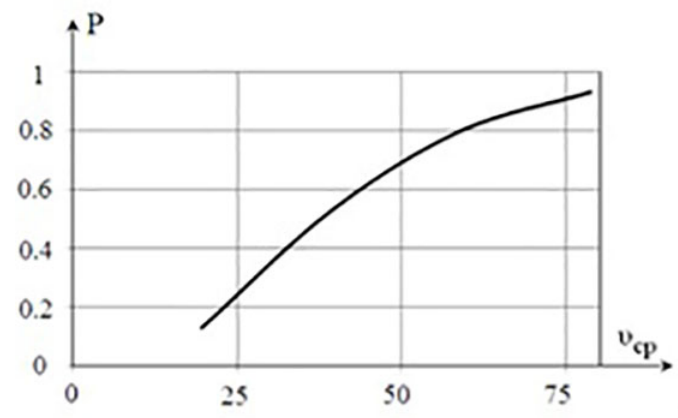

Fig. 2. Electrical contact failure probability

We believe that the considered issues of dust concentration impact on electrical contact operation is of a certain interest for reliable electrical networks designers. The suggested mathematical model enables to choose the basic parameters of switching elements considering potential contamination, and to develop new and clarify existing fault determination methods.

\section{Conclusion}

- A method for determination of transition resistance of low-level electrical contacts in dusty conditions is suggested.

- At dust concentration up to 20 particles per $\mathrm{mm}^{2}$ the failure probability may reach $15 \%$.

\section{References}

1. R. Holm, Electrical Contact (New York: Springer, 1979)

2. W. Merle, Electrical Contact (M.-L.: Gosenergoizdat, 1962)

3. J.A. Greenwood, Constriction resistance and the real area of contact, Brit. J. Appl. Physics, v. 17, pp. 1621-163 (1966)

4. J.A. Greenwood, J.B. Williamson, Electrical conduction in solids. II. Theory of temperature-dependent conductors, Proc. Roy. Soc., v. A246, pp.13-31 (1958)

5. B.S. Sotskov, Design and Calculation Foundations of Electromechanical Elements, Automated and Telemechanical Devices (M. -L.: Energiya, 1965)

6. A.P. Levin, Contacts of electrical connectors for radioelectronic equipment (M.: Sov. radio, 1972)

7. N.K. Myshkin, V.V. Konchits, M. Braunovich, Electrical Contacts (Dolgoprudny: Intellect, 2008)

8. E.S. Lyuminarskaja, V.N. Dianov, Mathematical model of low-level electrical contacts in case of failure, Dynamics of complex systems - XXI century, v. 8, pp. 73-78 (2014)

9. V.V. Izmailov, M.V.Novoselova, Contact of solids and conductivity: monograph (Tver: Tver State Technical University, 2010)

10. N.B. Demkin, Rough surface contacting (M.: Nauka, 1970) 
11. N.B. Demkin, M. A. Korotkov, Contact resistance of rough surfaces (M.: Nauka, 1972)

12. N.B. Demkin, E. V. Ryzhov, The surface quality and contact of machine parts (M.: Mechanical Engineering, 1981)

13. I. E. Andreev, Development and research of analytical and simulation models of contact switching systems: the abstract Diss. Cand. tech. Sciences (Kharkov, 1984)

14. R.S Timsit, Electrical contact resistance: Fundamental principles /Slade, P.G., ed. Electrical contacts principles and application (New York: Marcel Dekker, 1999)

15. I. A. Ivanov, I. G. Kiselev, S. V. Urushev, Transient electrical contact resistance considering surface roughness, Electrical engineering, no. 10, pp.33-36, (2017)

16. A. N. Lugin, The theory of electric contacts in the aspect of electrical conductivity of the resistive films, News of higher educational institutions. Electronics, no.3, pp. 33-39 (2012)

17. M. V. Murashov, S. D. Panin, S. M. Klimov, Numerical modeling of the electrical conductivity of the contacts of rough bodies, Engineering and computer science, no. 1, pp. 189-200 (2015)

18. A.A. Efimenko, S.V. Merlan, The transitional contact resistance in electrical connection with flat pins, Technology and designing in electronic equipment, no.4, pp. 3-7 (2013)

19. A.G. Vikulov, D.G. Vikulov, The influence of surface nanofilms on the electric heat conductance of the contact metals, Engineering physics, no.10, pp. 26-43 (2011) 